Editorial

\title{
Psychophysiological and behavior evaluation of human performance
}

A great deal of current studies focuses on different aspects of the human organism. For this reason, a multidisciplinary approach is gaining interest due to the information provided allowing to better comprehension and prediction of complex phenomena, such as human behaviour. Understanding how modifications in physiological and psychological mechanisms can affect behaviour and its relationship with different professional and health areas represents a challenge for numerous research groups.

In the present special issue, researchers achieved to enhance the new and existing knowledge in these areas. Thus, we provide information regarding the latest research concerning the role of stress in drug addiction, revealing its fundamental role in order to understand this antisocial behavior. The way that stress affects personnel ability to operate during highly stressful situations, such as combat, can distort the psychophysiological responses and posing the risk of significant physical harm. This response is accentuated when soldiers must use the nuclear, biological and chemical equipment since in actual theatre of operations is a constant risk for deployed personnel. This situation can negatively affect both psychophysiological and fine motor skills, highlighting the need for a training program that includes the use of specific equipment. Nevertheless, these extreme psychophysiological responses are trainable. For example, high-intensity interval training is one of the most operative training methods for military populations. In addition, an important transference to professional performance has been revealed. Moreover, young male offenders presented specific deficiencies in the recognition of facial expressions due to disgust, probably due to failures in the integration of this emotion with the context.

In the health area, authors examined the potential benefits of physical activity to prevent excessive immune system activation, the effectiveness of physical exercise as a non-pharmacological tool to positively modulate age-related decline in cognitive function in older adults, as well as the importance of a structured and periodized muscular and pilates training program towards the enhancement of static balance in older women. In this line, the actual pandemic of obesity and peripheral metabolic alterations may negatively modulate gene transcription pathways that interplay to compromise neurotropic factors levels in the hippocampus, two primordial mediators of cognitive processes. To counterbalance the harmful scenario induced by obesity, physical exercise has been shown to be a non-pharmacological treatment reversible epigenetic alteration resulting in better cognitive outcomes.
Focused on psychophysiological relationships during sports events, the interception has been shown to influence self-regulation of effort and perceived exertion during exercise, but interoceptive accuracy did not influence cardiac autonomic modulation perceptual responses and performance at submaximal and maximal intensities during maximal incremental exercise. Specifically, for the aid area, the ergogenic effect of caffeine ingestion was revealed, as an improved $20 \mathrm{~km}$ cycling time trial performance and psychological responses in mentally fatigued cyclists was shown, despite the unaltered prefrontal cortex activation. In this line, swimming training along with curcumin supplementation presented an antioxidant effect on the heart tissue of rats during withdrawal of binge alcohol consumption.

Finally, in the sports area, the encephalogram modifications of chess players in decision-making processes in rapid and lightning chess games were found, showing how activity patterns differ between the rapid and the lightning games, highlighting that theta power might be related with chunks retrieval. Training level can also affect psychological responses during sports-related tasks, while these responses are probably not evident under non-competitive situations, also suggesting that competitive level athletes need more challenging activation stimulus to induce the desirable adaptations after a potentiation activity. The importance of the hour of training on sleep quality was also analyzed, presenting that higher sleep efficiency exist when athletes followed a morning versus an evening training, or the relationship between salivary alpha-amylase and neurobehavioral alertness during extended wakefulness, suggesting that this parameter can be used as a potential circadian marker.

All these findings allow us to enhance, from a multidisciplinary point of view, the knowledge regarding the psychophysiological and behavior patterns of human performance and provide evidence and practical applications that can help physicians, trainers, and academic staff.

Vicente Javier Clemente-Suárez ${ }^{\mathrm{a}, \mathrm{b}, *}$, Juan Pedro Fuentes ${ }^{\mathrm{c}}$ ${ }^{a}$ Universidad Europea de Madrid, Faculty of Sports Sciences, Madrid, Spain ${ }^{\mathrm{b}}$ Grupo de Investigación en Cultura, Educación y Sociedad, Universidad de la Costa, Barranquilla, Colombia ${ }^{\mathrm{c}}$ Faculty of Sport Science, University of Extremadura, Extremadura, Spain E-mail address: vicentejavier.clemente@universidadeuropea.es (V.J. Clemente-Suárez).

\footnotetext{
* Corresponding author at: Faculty of Sport Science, Universidad Europea de Madrid, Villaviciosa de Odón, Madrid, Spain.
} 